\title{
Transition radiation at radio frequencies from ultra-high energy neutrino-induced showers
}

\section{Pavel Motloch*, Paolo Privitera}

Kavli Institute for Cosmological Physics, Department of Physics, University of Chicago, Chicago, Illinois 60637, USA

E-mail: motloch@uchicago.edu, priviter@kicp.uchicago.edu

\section{Jaime Alvarez-Muñiz, Enrique Zas}

Departamento de Física de Partículas \& Instituto Galego de Física de Altas Enerxías, Universidade de Santiago de Compostela, 157282 Santiago de Compostela, Spain E-mail: jaime.alvarezmuniz@gmail.com, zas@fpaxp1.usc.es

\begin{abstract}
Detection of transition radiation from neutrino-induced showers escaping a dense medium is a promising technique which might be employed in future generations of ultra-high energy (UHE) neutrino detectors. Using the well-known Zas-Halzen-Stanev (ZHS) algorithm, we have computed the electric field created by showers crossing a dense medium-air interface and investigated the phenomenology of the emitted signal. Our calculations show that transition radiation is sizeable in a wide solid angle range with full coherence up to $\sim 1 \mathrm{GHz}$. These properties could allow the design of large aperture detectors with low signal threshold. The work reported here represents a stepping stone for future dedicated investigations of particular experimental setups.
\end{abstract}

The 34th International Cosmic Ray Conference,

30 July- 6 August, 2015

The Hague, The Netherlands

* Speaker. 


\section{Introduction}

Extraterrestrial neutrinos could play a huge role in helping resolve the question of the UHE cosmic ray (CR) origin because they are expected to be produced together with UHE CR at the sources or in the interactions of the highest energy cosmic rays with the cosmic microwave and infrared backgrounds and are not affected by the galactic magnetic fields. Recently, the IceCube collaboration was the first to detect extraterrestrial neutrinos with energies in the range from 100 $\mathrm{TeV}$ to several $\mathrm{PeV}$ [1]. Unfortunately, due to the low neutrino interaction probability within the observed IceCube volume, it is possible to detect only a handful of events each year. Searching for alternative experimental designs and detection techniques is thus very desirable.

In this work we explore the possibility of detecting extraterrestrial neutrinos through transition radiation (TR) [2,3], emitted by particles which cross a boundary between two media with different indices of refraction. Whenever a neutrino passes through Earth and interacts close to the surface boundary, some of the particles from the ensuing particle shower escape to the atmosphere. Because of the Askaryan effect [4], there is a net electric charge passing through the boundary, creating an upward-going transition radiation during the process. Because particle showers in dense media are rather compact, the resulting signal from such a situation can be expected to be coherent to very high frequencies of order $\sim 1 \mathrm{GHz}$. Detecting the emission at these frequencies has notable advantages, such as low cost of detectors and low background noise.

In this contribution we explain a general method of calculating transition radiation emitted by a shower crossing boundary between two media in a far-field limit, which could be of a direct practical interest for satellite or baloon detection $[5,6]$. We also describe and comment on the phenomenology of TR signal induced by such a crossing shower.

\section{Calculation - ZHS-TR algorithm}

Our method for calculating radio emission from particle showers which cross a boundary between two media is based on the ZHS algorithm [7, 8, 9] for the calculation of the electric field that is implemented in the ZHS Monte Carlo code for the simulation of electromagnetic showers in homogeneous media. We first briefly review the original code before we focus on changes made to account for the transition radiation.

The code accounts for bremsstrahlung, pair production, and the four interactions responsible for the development of the excess charge: Møller, Bhabha, Compton scattering and $e^{+} e^{-}$annihilation. Multiple elastic scattering according to Molière's theory and continuous ionization losses are also implemented. The tracks of all charged particles in a shower are divided into multiple straight sub-tracks with particles moving along these with constant velocities. The positions of the end points of these subtracks and the corresponding times are then used to compute the frequency components of the electric field. The resulting electric field in the observer's location is then given by the sum of contributions from individual tracks, taking into account the relative phase shift between different tracks because of their different starting point positions and time delays.

From Maxwell equations it can be shown that a charged particle moving with a uniform speed $\vec{v}$ in a homogeneous nonmagnetic medium between two fixed points produces an electric field $\vec{E}(t, \vec{x})$ 


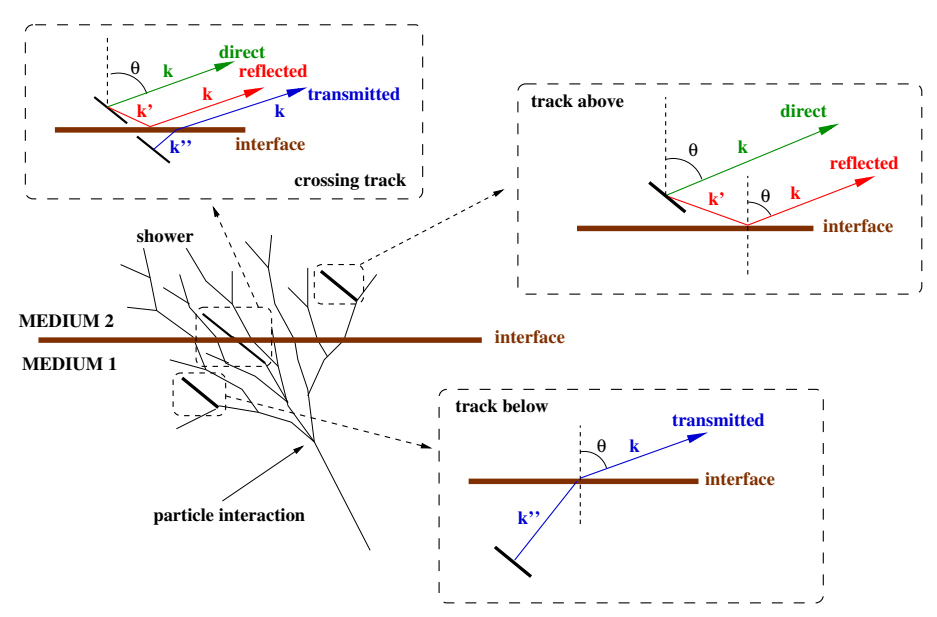

Figure 1: Schematic explanation of the ZHS-TR algorithm (see the text for description).

with frequency Fourier transform given by the expression [8]

$$
\vec{E}(\omega, \vec{x})=2 \int_{-\infty}^{\infty} \vec{E}(t, \vec{x}) e^{i \omega t} d t=\frac{e}{2 \pi \varepsilon_{0} c^{2}} i \omega \frac{e^{i k R}}{R} e^{i(\omega-\vec{k} \cdot \vec{v}) t_{1}} \vec{v}_{\perp}\left[\frac{e^{i(\omega-\vec{k} \cdot \vec{v}) \delta t}-1}{i(\omega-\vec{k} \cdot \vec{v})}\right] .
$$

Here $R$ is the distance from the track to the observer, assumed to be much larger than any other dimension in the situation. Wave vector $\vec{k}$ points in the direction of observation from the track to the observer and has modulus $k=\omega / c^{\prime}, c^{\prime}$ being the speed of light in the medium. In the formula, $\vec{v}_{\perp}=-\hat{k} \times(\hat{k} \times \vec{v})$ with the unit vector $\hat{k}=\vec{k} /|\vec{k}|$. Finally, $t_{1}$ and $t_{2}=t_{1}+\delta t$ are the absolute times at which the particle passes through each of the endpoints of the subtrack.

In our case, the presence of a boundary between two homogeneous media forces us to include several modifications to the original ZHS code. Our algorithm, which we call ZHS-TR, results from combining the ZHS algorithm with standard boundary matching techniques [10, 11, 12].

As before, we are interested in the calculation of the electric field observed far away from the shower in direction $\hat{k}$. Physical particle tracks are again approximated by line segments and it is assumed that particles move along the segments with constant velocity. The calculation of the contribution from an individual sub-track now depends on whether the given line segment is completely in one of the media or crosses the boundary. A pictorial explanation of the algorithm is presented in Fig. 1. For simplicity, only a planar boundary between the two media was considered.

For tracks entirely in the first medium where the shower starts, we calculate the electric field (2.1) emitted into direction $\hat{k}^{\prime \prime}$. This is the direction of a ray which is refracted on the boundary into the direction $\hat{k}$ according to the Snell's law. The radiated electric field is then decomposed into $s$ and $p$ polarizations and the electric field at the detector is determined using the Fresnel coefficients for transmission [10]. Because we are dealing with a point source and not plane waves, the usual transmission Fresnel coefficients must be multiplied by a factor which takes this difference into account and can be understood as describing the change in divergence of rays upon transmission [11]. When calculating the electric field at the detector, the $p$ polarization vector must be rotated to account for the change in the propagation direction upon transmission. Working in the Fraunhofer 
regime, in (2.1) we assume a constant attenuation factor $1 / R$ for all subtracks. In the following we refer to the electric field created by tracks in the first medium as the "transmitted contribution".

For tracks completely in the second medium, two distinct contributions must be combined. The first is the usual contribution (2.1) describing the electric field radiated into the direction $\hat{k}$ ("direct contribution"). We then have to add radiation which is reflected off the boundary ("reflected contribution"). Similarly to the previous case, we calculate radiation (2.1) emitted into direction $\hat{k}^{\prime}$ which is the direction of a ray which is reflected into the observing direction $\hat{k}$. We then perform analogous operations as for the transmitted contribution, without the extra multiplicative factor in the Fresnel coefficient as the signal propagates in the same medium before and after the reflection.

Finally, when there is a track which crosses the interface we split the track at the boundary into two subtracks, as in [11]. These are each exclusively in one or the other medium and can be accounted for in the manner explained in the previous paragraphs.

In the end, the electric field created by the whole shower is calculated as the superposition of contributions from all individual particle tracks. Care was taken to appropriately include explicit geometric phases between different contributions from various tracks on both sides of the boundary. Because of the limitations of the original code, we only account for purely electromagnetic showers. Also the geomagnetic emission from the shower and the height dependence of the air properties are neglected. However, neglecting these effects should not have a major impact on our results as we discuss in section $\S 3$.

We checked that our algorithm reproduces the exact analytical calculation - derived directly from the Maxwell's equations - of TR for an infinite track perpendicular to the boundary [12].

\section{Phenomenology}

In this section we investigate the electric field emitted by a particle shower leaving dense material and entering air. There was no thinning method employed in any of the presented calculations. We work in the Fraunhofer regime where the electric field $E(\omega, R, \hat{k})$ is inversely proportional to the distance from the shower $R$. For this reason we plot the magnitude of $R \times E$ in all graphs.

\subsection{Shower vertical with respect to the boundary}

The electric field created by a typical $100 \mathrm{TeV}$ vertical shower leaving ice (refractive index $\sim 1.78$ ) and entering air (r. i. 1.0003) is shown in Fig. 2. It is noticeable that unlike the coherent Cherenkov radiation which is strongly beamed around the Cherenkov angle [8], the transition radiation has a relatively weak angular dependence. The typical value of $|R \times E|$ for a $100 \mathrm{TeV}$ shower is $4-5 \times 10^{-7} \mathrm{~V} / \mathrm{MHz}$.

Figure 2 also shows magnitudes of the three contributions to the total electric field employed in our algorithm. Direct contribution from particles in air is the most siginificant component at all angles. This component is dominated by a peak at around $2^{\circ}$, which comes from forward-going particles in air. These propagate superluminally, each creating Cherenkov radiation beamed at $\sim 1.4^{\circ}$. As particles are mostly aligned with the shower axis direction, the total signal shows a peak at a similar angle. The reflected component is negligible except for the highest angles, where direct and reflected components are both radiated with similar intensities and nearly opposite phases, 


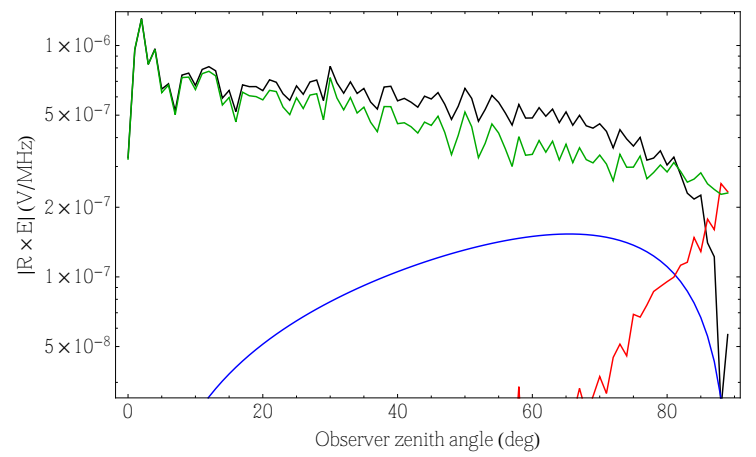

Figure 2: Angular distribution of the electric field radiated by a vertical $100 \mathrm{TeV} e^{-}$-induced shower going from ice to air; crossing at shower maximum. Decomposition of the total signal (black) into direct (green), reflected (red) and transmitted (blue) components (see Fig. 1). Signal at $200 \mathrm{MHz}$.

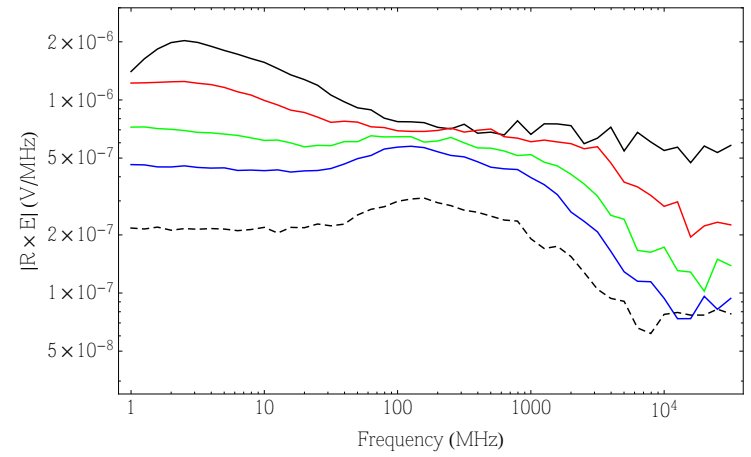

Figure 3: Frequency spectrum of the radiated electromagnetic pulse for several observer directions. Same shower configuration. Zenith angles of observers (see Fig. 1) are $10^{\circ}$ (black solid), $20^{\circ}$ (red), $40^{\circ}$ (green), $60^{\circ}$ (blue), $80^{\circ}$ (black dashed). Each curve is an average of 10 showers.

which leads to a drop of the signal around $90^{\circ}$ zenith angles. For a vertical shower going from ice to air, the contribution from particles in ice is never dominant.

The frequency dependence of the signal magnitude for several fixed observer directions is plotted in Fig. 3. Unlike the coherent Cherenkov radiation which drops off at relatively low frequencies once we go away from the Cherenkov angle [8], transition radiation signal is coherent up to $\sim 1 \mathrm{GHz}$ in a wide range of angles.

The TR signal from a vertical shower is mostly determined by the particle tracks crossing the interface. At frequencies below a cutoff frequency determined by the geometrical size of the shower, all particles contribute coherently and the physical signal scales roughly as the number of particle crossings. For showers which cross the interface at their shower maximum this corresponds to electric field scaling roughly linearly with energy of the shower. We simulated several thinned $1 \mathrm{EeV}$ showers and found agreement with the expected linear scaling of the signal with the energy to within a factor of 2-3 when we compare results for $100 \mathrm{TeV}$ and $1 \mathrm{EeV}$ showers.

We also checked that the TR electric field is fairly insensitive to the presence of interactions in air, which means that even our simplified treatment which neglects the geomagnetic effect and height dependence of air properties should give a reasonably good estimate of the emission.

For the showers which cross the surface away from the shower maximum, the TR signal is weakened at zenith angles above $10^{\circ}$, qualitatively tracing the number of crossing particles. At zenith angles below $5^{\circ}$ there is a strong contribution from a Cherenkov signal of particles in air, suppressed in showers with larger portion of development happening in the dense material.

Guided by the possibility of particle shower detection we also investigated emission from TR for showers leaving other dense materials. For comparison, we simulated the same physical configuration, only ice was exchanged for salt or regolith. The difference is not very pronounced at $200 \mathrm{MHz}$ but at higher frequencies, salt and regolith gain a slight edge over ice. This is caused by the fact that while for ice $1 \mathrm{GHz}$ is a frequency at which the signal in ice at higher zenith angles starts to be depleted by the interference effects, this is not the case for the other two materials. For 
them, the Molière radius is about half the value for ice. As a consequence, in salt and regolith all the particles still add fully coherently at $1 \mathrm{GHz}$ and the signal is thus slightly stronger than in ice.

\subsection{Shower inclined with respect to the boundary}

While showers with close to vertical incidence could be potentially useful for detecting UHE neutrinos passing through a mountain range, the more likely use of TR technique would be to detect inclined showers induced by neutrinos passing through Earth and interacting inside Earth close to the surface. At the neutrino energies above $100 \mathrm{TeV}$, the Earth starts to be increasingly opaque for vertically going neutrinos [13] and the majority of the signal would thus come from inclined showers.

To provide insights into how the TR signal from such showers looks like, we have simulated inclined electron-induced showers crossing a boundary from ice to air. The shower energy was set to $100 \mathrm{TeV}$ and the geometry was chosen such that the shower axis crosses the boundary at shower maximum. The magnitude of the radiated electric field observed from such a shower is plotted in Fig. 4 for two showers with zenith angles $45^{\circ}$ and $75^{\circ}$. Each point in the plot represents a single observer direction; distance from the center of the plot is proportional to the observer's nadir angle while his azimuth determines the polar angle in the plane of the plot. The direction of the shower axis is marked by a cross. For each shower we plot $|R \times E|$ at 50, 200 and $1000 \mathrm{MHz}$.

It is clear that at low frequencies the radiated electric field is significant over a large portion of the sky even for a very inclined shower. The strength of the electric field is comparable to the values we observed for the case of a vertical shower. Centered on the shower axis there is a small region of increased signal which comes from emission close to the Cherenkov cone for the particles in air. The magnitude of the electric field in this region is increased by about a factor of 3.

At higher frequencies, the portion of the hemisphere illuminated by a high value of the electric field decreases. For a shower with $45^{\circ}$ zenith angle this effect is not much pronounced even at $1 \mathrm{GHz}$. However, for the highly inclined shower with $75^{\circ}$ zenith angle the fraction of the hemisphere with $|R \times E|$ above $10^{-6.5} \mathrm{~V} / \mathrm{MHz}$ drops from $\sim 50 \%$ at $50 \mathrm{MHz}$ to $\sim 20 \%$ at $1 \mathrm{GHz}$. This makes detection of inclined showers more difficult at higher frequencies. On the other hand, at higher frequencies there appear regions with increased magnitudes of the radiated signal. Hints of this are visible already at $200 \mathrm{MHz}$, while at $1 \mathrm{GHz}$ we gain about an extra order of magnitude in signal strength for both showers. This gain is easily explained in terms of the Cherenkov radiation refracted from ice into air. Indeed the majority of the signal in these regions is coming from below the interface ("transmitted contribution") and we have verified the position of the Cherenkov cone with a simple geometrical model: the position of the maximal signal agrees with the position of the Cherenkov cone for a particle moving with the speed of light in ice along the shower axis, refracted into air according to the Snell's law. For the vertical shower, the signal in the direction of the Cherenkov cone in ice is totally internally reflected off the boundary and can not be detected by an observer in air.

\section{Conclusions}

We describe an algorithm, based on a extension of the well-known ZHS algorithm, to calculate in detail the radiation pattern induced by a shower crossing a boundary between two media, taking 

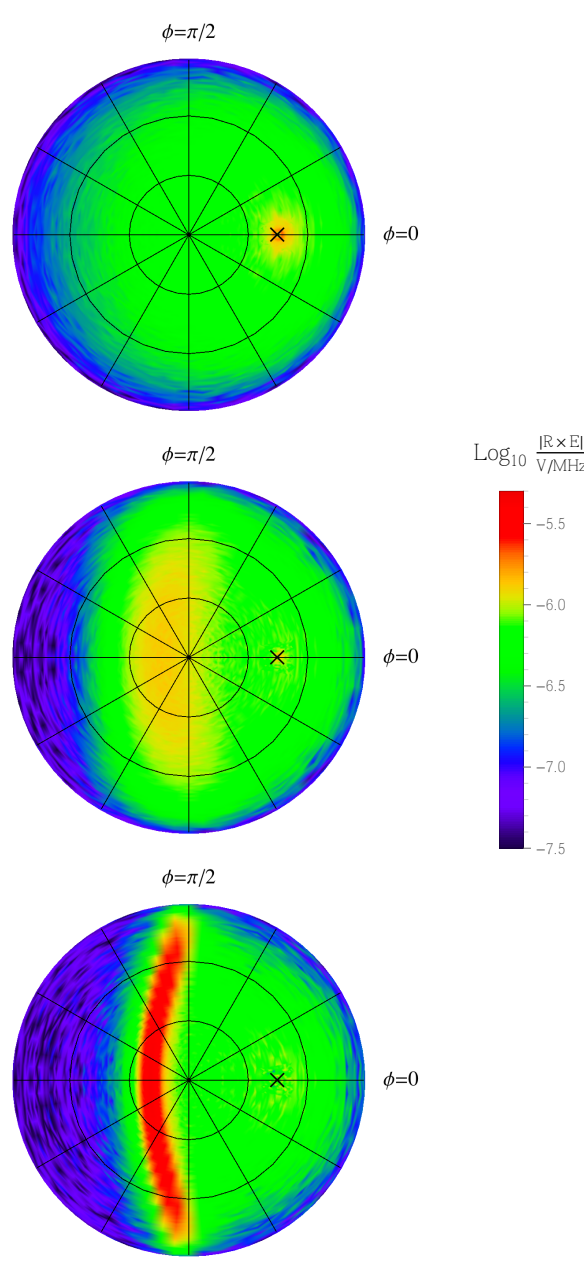
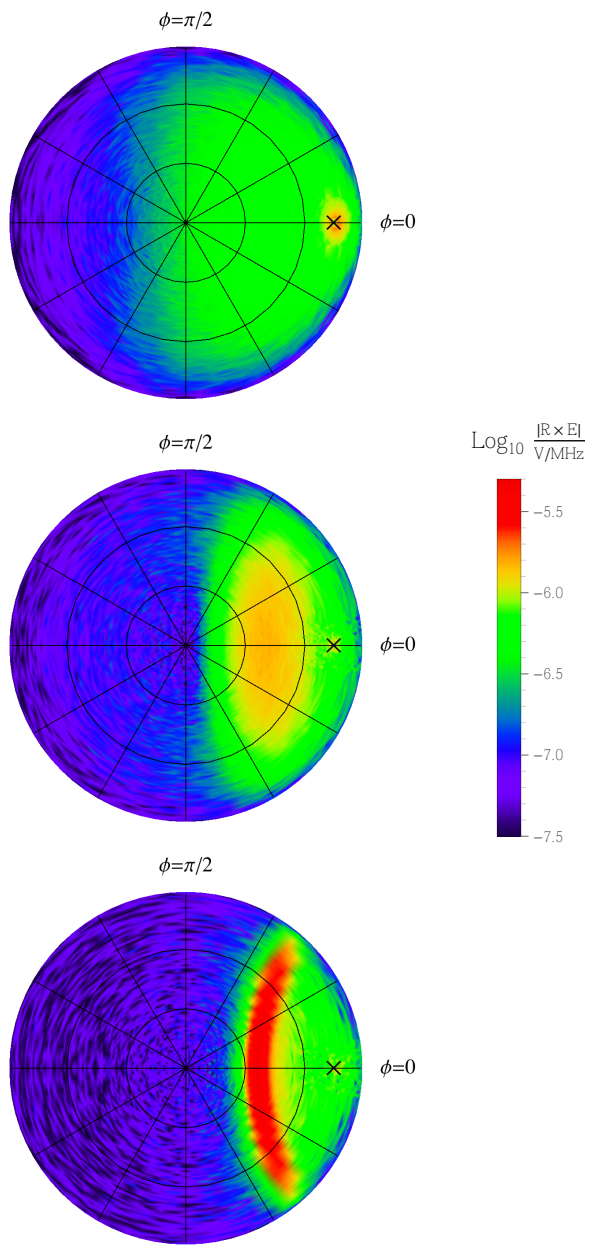

Figure 4: Dependence of the strength of a radio emission from an inclined $100 \mathrm{TeV}$ shower with zenith angle $45^{\circ}$ (left) and $75^{\circ}$ (right), leaving ice into air while crossing at shower maximum. Distance from the center of the plot stands for observer's zenith angle (the circles represent observers at $30^{\circ}$ and $60^{\circ}$ zenith angles), his azimuth $\phi$ determines the polar angle in the plot. Shower direction is indicated by the cross. Top figure at frequency $50 \mathrm{MHz}$, middle at $200 \mathrm{MHz}$, bottom at $1 \mathrm{GHz}$.

into account the coherent emission. The algorithm is based on approximating the charged particle tracks with linear segments and adding the contributions from all segments. Due to the presence of the boundary it is necessary to split crossing tracks into subtracks wholly contained within each medium and sum the direct, reflected and refracted contributions induced by each subtrack at either side of the boundary. We use this approach to investigate the transition radiation when the induced particle showers cross the boundary between Earth and air which could be of interest for the detection of extraterrestrial neutrinos.

We explore the phenomenology of such showers in the far-field limit. The signal for a vertical shower is fairly isotropic, with electric field of order $5 \times 10^{-11} \mathrm{~V} / \mathrm{MHz} / \mathrm{m}$ for a $100 \mathrm{TeV}$ shower crossing the ice/air boundary at the shower maximum, with observer at distance $10 \mathrm{~km}$. For show- 
ers crossing the boundary away from the shower maximum the signal is lower in proportion to the number of crossing particles as for nearly vertical showers the signal is dominated by contributions from subtracks crossing the boundary. The electric field is approximately proportional to the shower energy, and remains coherent up to $\sim 1 \mathrm{GHz}$ in ice and to even higher frequencies in salt and regolith. The strength of emission does not depend strongly on the nature of the dense medium.

For inclined showers, we again see large portion of the arrival direction hemisphere illuminated by a strong signal. At high shower inclinations the fraction with this enhanced signal diminishes with increasing frequency. At frequencies around $1 \mathrm{GHz}$ we see a big increase of the signal strength in well defined directions. This is due to strong electric field radiated in ice into the Cherenkov cone direction which is refracted at the boundary into the air.

Our calculations were done only for electromagnetic showers, such as those appearing in a charged-current interaction of a high-energy electron neutrino. However, we expect that our results are also useful in estimates of the TR radiation emitted by hadronic showers such as those produced in charged or neutral-current interactions of neutrinos of any flavor as a large fraction of the energy in these showers is eventually transferred into the electromagnetic component.

\section{Acknowledgements}

This work was supported in part by NSF grant PHY-1412261 and by the Kavli Institute for Cosmological Physics at the University of Chicago through NSF grants PHY-1125897, PHY0114422 and PHY-0551142 and an endowment from the Kavli Foundation and its founder Fred Kavli. JAM and EZ additionally thank Ministerio de Economía (FPA2012-39489), ConsoliderIngenio 2010 CPAN Programme (CSD2007-00042), Xunta de Galicia (GRC2013-024), Feder Funds and CESGA (Centro de Supercomputación de Galicia) for computing resources.

\section{References}

[1] M. G. Aartsen et al. [IceCube Collaboration], Science 342, 1242856 (2013).

[2] V. L. Ginzburg and I. M. Frank, J. Phys. (USSR) 9, 353 (1945).

[3] M. L. Ter-Mikaelian, High energy electromagnetic processes in condensed media, Wiley, New York, NY (1972).

[4] G. A. Askar'yan, Sov. Phys. JETP 14, no. 2, 441 (1962).

[5] P. W. Gorham et al. [ANITA Collaboration], Phys. Rev. D 82, 022004 (2010).

[6] P. Motloch, N. Hollon and P. Privitera, Astropart. Phys. 54, 40 (2014).

[7] H. R. Allan, Radio Emission from Extensive Air Showers, North-Holland, Amsterdam (1971).

[8] E. Zas, F. Halzen and T. Stanev, Phys. Rev. D 45, 362 (1992).

[9] D. Garcia-Fernandez et al., Phys. Rev. D 87, no. 2, 023003 (2013).

[10] J. D. Jackson, Classical electrodynamics, 3rd. ed., Wiley, New York, NY (1999).

[11] C. W. James, H. Falcke, T. Huege and M. Ludwig, Phys. Rev. A 84, 056602 (2011).

[12] V. L. Ginzburg and V. N. Tsytovich, Transition rad. and rad. scattering, Hilber, Bristol, UK (1990).

[13] R. Gandhi, C. Quigg, M. H. Reno and I. Sarcevic, Phys. Rev. D 58, 093009 (1998). 